\title{
Psychotropic drugs for the behavioural and psychological symptoms of dementia: no free ride
}

\author{
Elia Abi-Jaoude MSc MD, Nathan M. Stall MD, Paula A. Rochon MD MPH
}

n Cite as: CMAJ 2018 November 26;190:E1374-5. doi: 10.1503/cmaj.181486

See related article at www.cmaj.ca/lookup/doi/10.1503/cmaj.180551

$\mathbf{N}$ early $70 \%$ of long-term care residents in Canada have dementia, and, at some point, most experience associated behavioural and psychological symptoms, which include disordered perception, thought content, mood or behaviour. ${ }^{1}$ Behavioural and psychological symptoms of dementia are among the most challenging and stressful aspects of caring for people with dementia. ${ }^{2}$ Despite only modest evidence of benefit, antipsychotics are commonly prescribed to older adults with dementia, including nearly $40 \%$ of the Canadian population living in long-term care who had at least 1 claim for an antipsychotic in 2014. ${ }^{3,4}$ Since the early 2000s, concerns have emerged about the safety of these agents, such as an increased risk of death and cerebrovascular events among adults with dementia. ${ }^{5,6}$ This evidence prompted warnings from regulatory agencies and widespread efforts for more judicious prescribing of antipsychotics to older adults with dementia. ${ }^{7}$ Trazodone has been increasingly used as an alternative option for the management of behavioural and psychological symptoms of dementia, despite inconclusive evidence about its benefit. ${ }^{8}$ An important question is whether trazodone is a safer alternative to antipsychotics.

In a linked retrospective propensity score-matched cohort study, Watt and colleagues used linked health administrative data to compare the risk of harm associated with trazodone to that of 3 of the most commonly used atypical antipsychotics (risperidone, olanzapine and quetiapine) among older long-term care residents with dementia. ${ }^{9}$ The study included 6588 older adults newly dispensed trazodone, and 2875 newly dispensed an antipsychotic. Compared with use of antipsychotics, use of trazodone was associated with a similar rate of the primary composite outcome of falls and major osteoporotic fractures within 90 days of the index prescription. Trazodone was associated with a lower mortality rate than antipsychotics, although a secondary outcome.

These timely findings show that trazodone is not a universally safer alternative to antipsychotics for the management of behavioural and psychological symptoms of dementia. Another recent study showed that new use of trazodone and benzodiazepines was associated with comparable risks of fall-related injuries among long-term care residents. ${ }^{10}$ As campaigns such as Choosing Wisely continue efforts to reduce antipsychotic use among

\section{KEY POINTS}

- Antipsychotics are commonly prescribed for the behavioural and psychological symptoms of dementia, despite only modest evidence for benefit and well-recognized serious risks.

- Trazodone has been increasingly used as an alternative option, yet accumulating evidence suggests that trazodone is not without risk.

- Behavioural, environmental and caregiver interventions show greater effect than many psychotropic drugs for the management of behavioural and psychological symptoms of dementia, and should be prioritized.

- Prescribing of psychotropic drugs for these symptoms should follow the geriatric medicine principle of "start low, and go slow"; there is currently no universally safe drug option.

older adults with dementia, the linked study underscores concerns about collateral increases in the prescription of other psychotropic drugs. ${ }^{9}$

Watt and colleagues also underscore the importance of prioritizing nonpharmacological approaches for the management of behavioural and psychological symptoms of dementia. ${ }^{2}$ Nonpharmacological approaches comprise a variety of behavioural, environmental and caregiver-supportive interventions, and existing evidence suggests that these show greater effect than many psychotropic drug therapies. ${ }^{2}$ Nonpharmacological interventions should be individualized to the older adult with dementia. They can be grouped into interventions targeting the person with dementia, the caregiver and those addressing the environment.

Interventions targeting the person with dementia include attending to unmet needs, such as pain, sleep problems, fear, boredom, loss of control or purpose, and acute medical problems. Interventions targeting the caregiver include education about dementia, help with communication, and supports around stress management. Interventions targeting the environment include attending to over- or understimulation, providing activity, structure and routines, and addressing safety issues. ${ }^{2}$ It is also important to consider drugs that may impair cognition, especially for adults in long-term care, who are often prescribed multiple psychoactive medications. ${ }^{4}$ 
Psychotropic drugs can play a useful role in the management of behavioural and psychological symptoms of dementia (e.g., situations where the safety of the person with dementia or others is at risk).,11 A trial of drug therapy may also be warranted when nonpharmacological approaches alone are not sufficient and the behavioural and psychological symptoms of dementia are particularly distressing. ${ }^{12}$ If drug therapy is being considered, it is important for the clinician to determine whether the symptoms are likely to respond to a psychotropic drug. In particular, psychosis, physical aggression, anxiety and depression may improve, whereas other symptom clusters, such as resistance to care, pacing and exit-seeking, are unlikely to change. ${ }^{12}$ The potential benefits of pharmacological treatment must be weighed against the potential harms and informed consent obtained from the person with dementia or their substitute decision-maker. ${ }^{11}$

Prescribing of psychotropic drugs for behavioural and psychological symptoms of dementia should follow the geriatric medicine principle of "start low, and go slow"; that is, clinicians should prescribe a short course, and monitor symptoms and behaviour carefully, as well as adverse drug effects. Follow-up is critical, with frequent reassessment of the ongoing need for psychotropic drug therapy; if symptoms and behaviours have stabilized, or if the drug is ineffective, the prescriber should consider reducing the dose or, in some cases, discontinuing the medication. ${ }^{11,12}$

Numerous initiatives in Canada and elsewhere support deprescribing antipsychotics in the long-term care setting. Early results from the Canadian Foundation for Healthcare Improvement's Antipsychotic Reduction Collaborative suggest that dose reduction or discontinuation of antipsychotics in long-term care residents resulted in reduced rates of falls without exacerbation of behavioural and psychological symptoms of dementia. ${ }^{13}$

Overall, clinicians and caregivers managing the care of people with behavioural and psychological symptoms of dementia should recognize that there is currently no universally safe drug option. Health and long-term care systems must strive to better integrate the 3 groups of nonpharmacological interventions for the management of behavioural and psychological symptoms of dementia. We owe this to older adults with dementia, who are some of the most vulnerable members of our society.

\section{References}

1. Dementia in Canada. Ottawa: Canadian Institute for Health Information. Available: www.cihi.ca/en/dementia-in-canada (accessed 2018 Oct. 11).

2. Kales HC, Gitlin LN, Lyketsos CG. Assessment and management of behavioral and psychological symptoms of dementia. BMJ 2015;350:h369.

3. Ballard C, Waite J. The effectiveness of atypical antipsychotics for the treatment of aggression and psychosis in Alzheimer's disease. Cochrane Database Syst Rev 2006;(1):CD003476.

4. Use of antipsychotics among seniors living in long-term care facilities, 2014 [report]. Ottawa: Canadian Institute for Health Information; 2016. Available: https://secure. cihi.ca/free_products/LTC_AiB_v2_19_EN_web.pdf (accessed 2018 Nov. 7).

5. Gill SS, Bronskill SE, Normand SL, et al. Antipsychotic drug use and mortality in older adults with dementia. Ann Intern Med 2007;146:775-86.

6. Rochon PA, Normand SL, Gomes T, et al. Antipsychotic therapy and short-term serious events in older adults with dementia. Arch Intern Med 2008;168:1090-6.

7. Lenzer J. FDA warns about using antipsychotic drugs for dementia. BMJ 2005;330:922.

8. Iaboni A, Bronskill SE, Reynolds KB, et al. Changing pattern of sedative use in older adults: a population-based cohort study. Drugs Aging 2016;33:523-33.

9. Watt JA, Gomes T, Bronskill SE, et al. Comparative risk of harm associated with trazodone or atypical antipsychotic use in older adults with dementia: a retrospective cohort study. CMAJ 2018;190:E1376-83.

10. Bronskill SE, Campitelli MA, laboni A, et al. Low-dose trazodone, benzodiazepines, and fall-related injuries in nursing homes: a matched-cohort study. J Am Geriatr Soc 2018;66:1963-71.

11. Reus VI, Fochtmann LJ, Eyler AE, et al. The American Psychiatric Association practice guideline on the use of antipsychotics to treat agitation or psychosis in patients with dementia. Am J Psychiatry 2016;173:543-6.

12. Use of antipsychotics in behavioural and psychological symptoms of dementia (BPSD) discussion guide. Long-Term Care (LTC 2nd ed). Toronto: Centre for Effective Practice; 2016. Available: https://thewellhealth.ca/wp-content/uploads /2016/05/UseofAntipsychotics_LTC2016-2.pdf (accessed 2018 Nov. 8).

13. Antipsychotic reduction collaborative results. Ottawa: Canadian Foundation for Healthcare Improvement. Available: www.cfhi-fcass.ca/WhatWeDo/recent -programs/reducing-antipsychotic-medication-use-collaborative/results (accessed 2018 Nov. 8).
Competing interests: Paula Rochon and Nathan Stall have connections to the authors of the linked study; neither had any involvement in the study. Paula Rochon and Nathan Stall have collaborated with the study authors on a series of research projects; Paula Rochon has published with or held grants with Tara Gomes, Susan Bronskill, Peter Austin, Joanne Ho and Sharon Straus; Susan Bronskill is on Nathan Stall's thesis committee, and they have published together. The authors have no financial conflict of interest related to this project. Paula Rochon holds The Retired Teachers of Ontario / Les enseignantes et enseignants retraités de l'Ontario Chair in Geriatric Medicine at the University of Toronto. No other competing interests were declared.

This article was solicited and has not been peer reviewed.

Affiliations: Department of Psychiatry (Abi-Jaoude), The Hospital for Sick Children; Department of Psychiatry (Abi-Jaoude), Toronto Western Hospital, University Health Network; Division of Geriatric Medicine
(Stall, Rochon), Department of Medicine, and Institute of Health Policy, Management and Evaluation (Stall, Rochon), University of Toronto; Women's College Research Institute (Stall, Rochon), Women's College Hospital, Toronto, Ont.

Contributors: All authors contributed to the interpretation of the linked study and provided input regarding the planned content of the commentary. Elia Abi-Jaoude wrote the first draft; Nathan Stall and Paula Rochon made substantial revisions. All authors gave final approval of the version to be published and agreed to be accountable for all aspects of the work.

Disclaimer: Nathan Stall serves on the editorial advisory board of CMAJ and was not involved in the editorial decision-making process for this article.

Correspondence to: Elia Abi-Jaoude, elia.abi.jaoude@utoronto.ca 